\title{
nature
}

\section{Malaria after the genome}

Following the publication of the malaria parasite's genome sequence and the beginnings of relevant proteomics, research tools are now available that could make a big difference in the long-term war against malaria.

$\mathrm{T}$ uberculosis. AIDS. Malaria. The three great modern plagues of the world's poor. The battle against them now stands at a crucial juncture. A committed effort by governments will determine whether, or how soon, victory is to be had. The United States in particular has a key role to play in shaping the current transformation in the way the world deals with international health, in the largest shift since the World Health Organization (WHO) was created as part of the United Nations after the Second World War.

The WHO retains its key role as the broker of intergovernmental relations between the health ministries of 192 countries. But over the past decade, a global reawakening of consciousness that these scourges can and must be defeated has spawned a plethora of new international players. These include collaborations between the public and private sectors, initiatives by charities, and the Global Fund to Fight AIDS, Tuberculosis and Malaria, which is yet to prove itselfbut is perhaps the first realistic effort to control these diseases using existing tools.

The United States should bring leadership to this movement. In particular, it should ensure that existing science is used effectively, by supporting control efforts through more generous support for bodies such as the Global Fund. It is widely agreed that a proper control programme for malaria would cost at least US $\$ 2$ billion annually - the equivalent of just two days' worth of US and European Union farm subsidies. It is less a question of money than of political will.

But existing science cannot on its own control malaria or other neglected diseases of the poor. We need science to yield new tools. In particular, there is a golden opportunity to engender solid progress in the fight against malaria, which every day kills between 2,500 and 8,000 children under five years of age. That we do not know how many children die says much about the lack of demographic and epidemiological research. One-sixth of the Earth's population suffers from the disease, crippling economic growth in those countries across South America, Africa and Southeast Asia that need it most. The challenges are made all the more urgent by the development of multidrug resistance by the parasite (see Nature 415, 670-672; 2002).

\section{On the map}

Science has now provided such an opportunity. Nature recently published the genome sequence of the malaria parasite Plasmodium falciparum, and with it, fundamental information that is critical to understanding the parasite. Whereas malaria researchers previously had to slog for months or years to find and study this or that gene or protein, a consortium of US and other researchers has now made maps of them all available to the world. And that for the modest sum of $\$ 15$ million over six years.

This is an important step along the way, and provides a new platform on which to develop essential insights into the many biomolecular and cellular pathways by which the parasite survives and interacts with us, its indispensable host. New techniques are beginning to be applied, such as high-throughput analyses of the pattern of gene expression and of the interactions of proteins at key phases of the parasite's life cycle. The availability of the genome combined with these techniques will undoubtedly spur progress significantly — if there are funds and the international structures to permit it.
A step towards the latter is the Multilateral Initiative on Malaria, created in 1997 to coordinate worldwide research, in large part thanks to the vision of Harold Varmus, then director of the US National Institutes of Health (NIH). The funds needed would not be enormous in absolute terms for the science that would flow from a post-genomics initiative. But they would be significant relative to the total world funding for malaria research, currently around $\$ 100$ million annually.

\section{International effort}

Inevitably, detractors will argue that post-genomics research is a luxury, and that money would be better spent on developing better drugs, or a vaccine, or on enhancing control of the disease through prevention. This misses the point. Funds for malaria research and drug and vaccine development are woefully inadequate (see Nature $419,426-428$; 2002). But history shows that every one of these alternative routes has its own chronic difficulties. Addressing new opportunities in the basic science of the disease should, in the long term, deliver far-reaching solutions. This should be funded by new money, increasing the pool of malaria research funds. It would therefore be widely recognized as a wonderfully enlightened action if the $\mathrm{NIH}$ were to take the lead in creating a substantial international malaria post-genomics programme.

An international effort would be money well spent. The availability of the genome has already stimulated lightning progress in deciphering the parasite's proteome by attracting to malaria research some of the brightest minds from the yeast community. There are many excellent researchers who would make rapid progress in malarial post-genomics if substantial new money were made available.

The malaria genome is already providing a service to the wider malaria research community. African scientists are using molecular markers of drug resistance, giving rise to country-wide 'early warning' maps; resistance was previously measured when it was too late, by the number of people who failed to respond to treatment. A postgenomics effort would yield valuable data and reagents for scientists in almost every area of malaria research.

Any new effort should pay particular attention to dissemination, through workshops, training and online databases, in particular to benefit scientists in Africa, where nine out of ten deaths from malaria occur. It also needs to guard against becoming a machine solely for generating scientific papers, disconnected from the wider war on the disease. Malaria post-genomics should not be an island, but should be as inclusive as possible. It should also be firmly anchored to existing nascent initiatives to ensure as much cross-fertilization as possible among areas of research.

For the United States to provide significant new help in this battle would not simply represent an act of great goodwill. It would also be in the nation's long-term strategic interests. Reducing developing countries' battle with the illness and mortality of malaria and the social burdens that they bring would allow them to focus more on economic and social development, providing new opportunities for US businesses and other organizations. It's over 30 years since humans set foot on the Moon; the time has come for a decade-long initiative towards freeing mankind from malaria. 